\title{
Time-resolved spectroscopic diagnostic of laser-induced plasma on germanium targets
}

\author{
J. J. Camacho, ${ }^{1, a)}$ L. Diaz, ${ }^{2}$ and J. M. L. Poyato ${ }^{1}$ \\ ${ }^{1}$ Departamento de Química-Física Aplicada, Facultad de Ciencias, Universidad Autónoma de Madrid, \\ Cantoblanco, 28049-Madrid, Spain \\ ${ }^{2}$ Instituto de Estructura de la Materia, CFMAC, CSIC, Serrano 121, 28006-Madrid, Spain
}

(Received 19 January 2011; accepted 9 April 2011; published online 20 May 2011)

Time-resolved optical emission measurements have been made in laser-induced plasma plumes following $\mathrm{CO}_{2}$ pulsed-laser $\left(10.591 \mu \mathrm{m}, 64 \mathrm{~ns}\right.$ and intensity from 0.27 to $\left.4.9 \mathrm{GW} \mathrm{cm}^{-2}\right)$ irradiation of germanium targets. The temporal behavior of $\mathrm{Ge}$ atoms and ionized excited species $\mathrm{Ge}^{3+}, \mathrm{Ge}^{2+}$, and $\mathrm{Ge}^{+}$is reported. The results show a faster decay of the continuum emission and $\mathrm{Ge}^{3+}, \mathrm{Ge}^{2+}$ ionic species than in the case of $\mathrm{Ge}^{+}$and neutral $\mathrm{Ge}$ atoms. The velocity distributions for $\mathrm{Ge}^{3+}$, $\mathrm{Ge}^{2+}, \mathrm{Ge}^{+}$, and $\mathrm{Ge}$ species are obtained from time-of-flight measurements. Electron density in the laser-induced plasma was estimated from the analysis of spectral data at various times from the $\mathrm{CO}_{2}$ laser pulse incidence. From the intensity decay with the delay time for $\mathrm{Ge}^{+}, \mathrm{Ge}^{2+}$, and $\mathrm{Ge}^{3+}$, we estimated the three-body electron-ion recombination rate constants for these species. (C) 2011 American Institute of Physics. [doi:10.1063/1.3590159]

\section{INTRODUCTION}

Germanium is an important semiconductor material used in electronic devices, fiber-optic systems, infrared optics, semiconductor-based detectors, and polymerization catalysts. It is also used as an alloying agent to increase the refraction index of glasses or as substrate wafers for highefficiency multi-junction solar cells. ${ }^{1}$ It is well known that germanium under normal absorption conditions is transparent to IR radiation. However, when the laser intensity increases and reaches a certain threshold value, as happens with high-power lasers, Ge is no longer transparent. The dynamics of materials ablated from surfaces by pulsed-laser irradiation are of fundamental technological and physical interest. The interaction of a pulsed high-power laser beam with matter and the consequent plasma production has been studied for many years. ${ }^{2-4}$ In particular the plasma induced by nanosecond lasers is successfully employed for Ge thin film deposition. ${ }^{5,6}$ The interest in laser-induced breakdown (LIB) is due to a number of potential applications of high electron density and high-temperature plasmas. ${ }^{7,8}$ Because of the transient characteristics of the plasma plume created by LIB, optical emission spectroscopy (OES) technique with time and space resolution is especially appropriate to obtain information about the behavior of the formed species as well as to study the dynamics of the plasma expansion. The temporally resolved LIB technique in Ge has been studied by several researchers ${ }^{9,10}$ using high-power lasers. Detailed studies of LIB plasmas in different samples have been reported recently by us. ${ }^{11-15}$

The present paper is aimed at discussing thermo-chemical processes induced by high-power IR $\mathrm{CO}_{2}$ pulsed laser on germanium targets. A spectroscopic study of Ge plasma, initially at room temperature, produced by high-power $\mathrm{CO}_{2}$ laser has

\footnotetext{
a) Author to whom correspondence should be addressed. Electronic mail: j.j.camacho@uam.es.
}

been carried out in an attempt to clarify the processes and the mechanism responsible for the emission. The emission observed in the plasma region is mainly due to electronic relaxation of excited $\mathrm{Ge}, \mathrm{Ge}^{+}, \mathrm{Ge}^{2+}$, and $\mathrm{Ge}^{3+}$ fragments. We present new results obtained from the time-resolved spectroscopic analysis. The velocity distributions for the electronically excited species are obtained from time-of-flight (TOF) measurements using time-resolved OES. Intensities of some lines from $\mathrm{Ge}$ and $\mathrm{Ge}^{3+}$ were used for determining electron temperature and their Stark-broadened profiles were employed to calculate electron density. The temporal behavior of intensity lines of $\mathrm{Ge}^{+}, \mathrm{Ge}^{2+}$, and $\mathrm{Ge}^{3+}$ has been employed for the estimation of the three-body electron-ion recombination rate constants for these species.

\section{EXPERIMENTAL PROCEDURES}

The experimental setup (Fig. 1) and equipment used have been described elsewhere. ${ }^{15}$ The experiments were carried out with a transversely excited atmospheric (TEA) $\mathrm{CO}_{2}$ pulsed-laser with a pulse intensity of $0.27-4.9 \mathrm{GW} \mathrm{cm}^{-2}$ at a wavelength of $10.591 \mu \mathrm{m}$. The laser-pulse intensity was varied with the aid of several calibrated $\mathrm{CaF}_{2}$ attenuating plates. The temporal shape of the laser pulse is shown in Fig. 2. The pulsed laser light was focused by a $\mathrm{NaCl}$ lens of $24 \mathrm{~cm}$ focal length onto a Ge polished crystalline target (Crystran) at medium vacuum conditions $\left(P_{\text {air }}=4 \mathrm{~Pa}\right)$. The measured focused-spot area was $7.85 \times 10^{-3} \mathrm{~cm}^{2}$. The optical emission from the laser induced plasma was imaged $1: 1$ by a quartz lens (focal length $4 \mathrm{~cm}$ ) onto the entrance slit of a spectrometer with a resolution of $\approx 0.1 \AA$ in first order. The Ge plasma emission was collected in a direction perpendicular to the incident laser beam at a fixed distance $z$ along the plasma axis. To change the $z$ distance, the target and the focusing $\mathrm{NaCl}$ lens were displaced to obtain a minimum focal spot area. Taking into account the size of the spectrograph slit (10 $\mathrm{mm}, 0.01 \mathrm{~mm})$, the inspection volume is quite small 


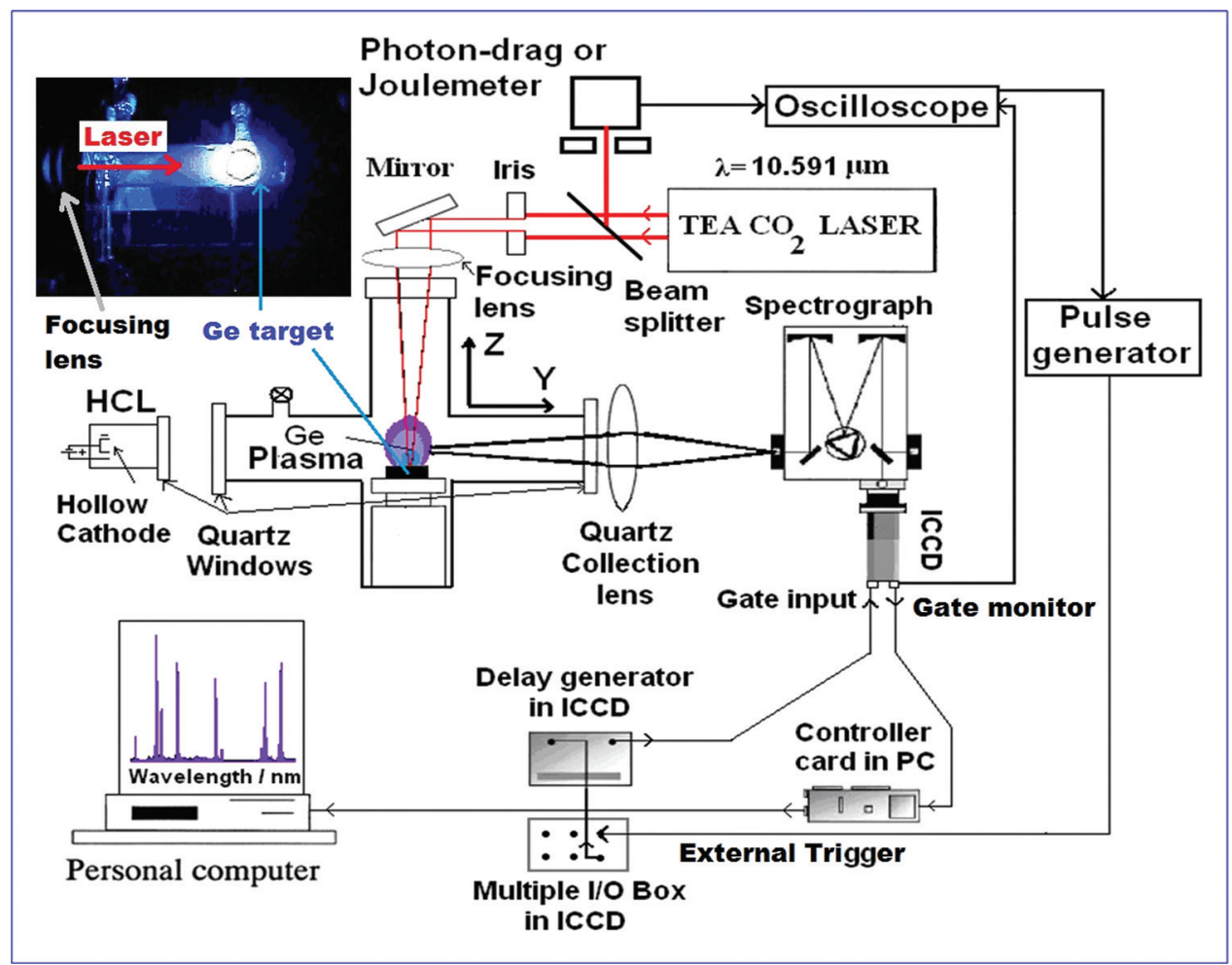

FIG. 1. (Color online) Schematic diagram of the experimental setup for the time-resolved spectroscopic measurements of the TEA-CO ${ }_{2}$ LIB Ge plasma.

compared to the total volume of the plasma. The spectra were recorded by a gateable intensified charge-coupled device (ICCD, Andor iStar DH-734). For time-resolved measurements the ICCD detector is synchronized with the trigger of the laser pulse adjusting a gate width time $t_{\mathrm{w}}$ and the delay time $t_{\mathrm{d}}$. Several hollow cathode lamps (HCL) were used for the spectral wavelength calibration of the spectrometer. The intensity response of the detection system was calibrated with a standard halogen lamp.

\section{RESULTS AND DISCUSSION}

When a high-power laser pulse is focused on a solid surface, the target becomes ablated and there is a breakdown

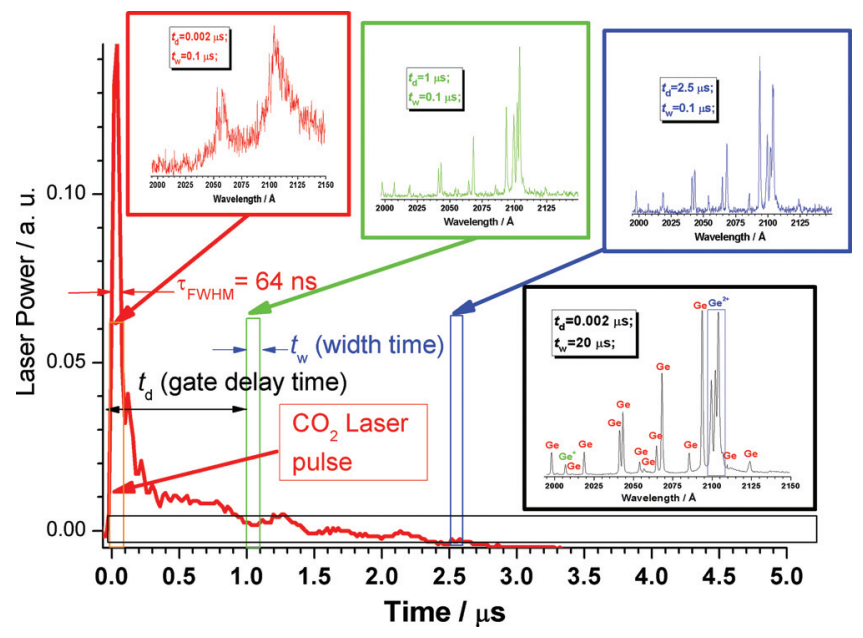

FIG. 2. (Color online) A schematic overview of the temporal history of the LIB Ge plasma. The temporal shape of the laser pulse is shown. Inset plots illustrate some spectra observed at different delay and width times. characterized by a brilliant flash of light accompanied by a distinctive cracking noise. At the top of Fig. 1, we show an image of the LIB plasma in Ge induced by a single $\mathrm{CO}_{2}$ laser pulse. The temporal history of the LIB Ge plasma is illustrated schematically in Fig. 2. The time for the beginning of the laser pulse is considered as the origin of the time scale. The inset pictures illustrate some emission spectra recorded at different delay $t_{\mathrm{d}}$ and width $t_{\mathrm{w}}$ times. For short delays, the continuum emission significantly contributes to the total emission.

To understand the detailed aspects of laser-beam interaction with $\mathrm{Ge}$ and recombination processes following the breakdown, OES studies from the plasma offer the most convenient technique. The LIB emission spectra of Ge show atomic-ionic lines from $\mathrm{Ge}, \mathrm{Ge}^{+} ; \mathrm{Ge}^{2+}$, and $\mathrm{Ge}^{3+}$. Strong ionic $\mathrm{Ge}^{3+}$ lines dominate the spectrum indicating the high ionization of germanium produced by LIB.

The LIB spectra of Ge were measured at different delay and width times. In a first set of temporal measurements, the spectra had been obtained in the spectral region 2000-2150 $\AA$, at a delay time ranging from 0.002 to $5 \mu$ s and $z=15 \mathrm{~mm}$ (Fig. 3(a)). Also shown in this figure is the time-integrated spectrum for $t_{\mathrm{d}}=0.002 \mu \mathrm{s}$ and $t_{\mathrm{w}}=20 \mu \mathrm{s}$. The spectral range was chosen to detect atomic, single, and double ionized $\mathrm{Ge}$ species. During the initial stages after the laser pulse $\left(t_{\mathrm{d}} \leq 1\right.$ $\mu \mathrm{s}), \mathrm{Ge}^{2+}$ emission dominates the spectrum. The emission lines for $\mathrm{Ge}^{2+}$ become progressively and spectrally narrower as a consequence of the variation in electron number density. This indicates that the electron density and excitation temperature decrease during the plasma expansion. When the delay is increased $\left(t_{\mathrm{d}} \geq 1 \mu \mathrm{s}\right)$, the intensity of $\mathrm{Ge}^{2+}$ ionic lines steeply decreases as a consequence of the radiative 
recombination between ions and electrons being detected up to $\approx 4 \mu \mathrm{s}$. For $t_{\mathrm{d}} \leq 0.2 \mu \mathrm{s}$, no apparent $\mathrm{Ge}$ emission was observed. When the delay is increased $\left(t_{\mathrm{d}} \geq 0.2 \mu \mathrm{s}\right)$, the intensity of $\mathrm{Ge}$ atomic lines steeply increases, also due to the electron-ion recombination in the plasma. The intensities of Ge lines reach the maximum at around $1.5 \mu \mathrm{s}$ and are detected up to $\approx 5 \mu \mathrm{s}$. Figure 3(b) displays a similar temporal evolution of the LIB spectrum of germanium plasma in the spectral region $2425-2570 \AA$ at delay times ranging from 0.002 to $2.5 \mu \mathrm{s}$ and $z=2 \mathrm{~mm}$. Also shown is the timeintegrated spectrum for $t_{\mathrm{d}}=0.002 \mu \mathrm{s}$ and $t_{\mathrm{w}}=10 \mu \mathrm{s}$. The spectral range was chosen to detect $\mathrm{Ge}, \mathrm{Ge}^{+}$, and $\mathrm{Ge}^{3+}$ species. In spite of our use of high-purity germanium, emissions from impurities, especially from nitrogen species as well as fragments of dissociated water molecules $\left(\mathrm{H}_{2}\right.$ and $\left.\mathrm{OH}\right)$, were observed. During the initial stages after the laser pulse $\left(t_{\mathrm{d}} \leq 2.5 \mu \mathrm{s}\right), \mathrm{Ge}^{3+}$ emissions dominate the spectra. The intensities for the emission lines of $\mathrm{Ge}^{3+}$ show a maximum at $0.1 \mu \mathrm{s}$. As in the preceding text, the emission lines for $\mathrm{Ge}^{3+}$ for $t_{\mathrm{d}} \geq 0.1 \mu \mathrm{s}$ become progressively and spectrally narrower as a consequence of the electron number density variation. The Ge emission lines, due to the actual observation distance used $(z=2 \mathrm{~mm})$, were now observed during the initial stages after the laser pulse.
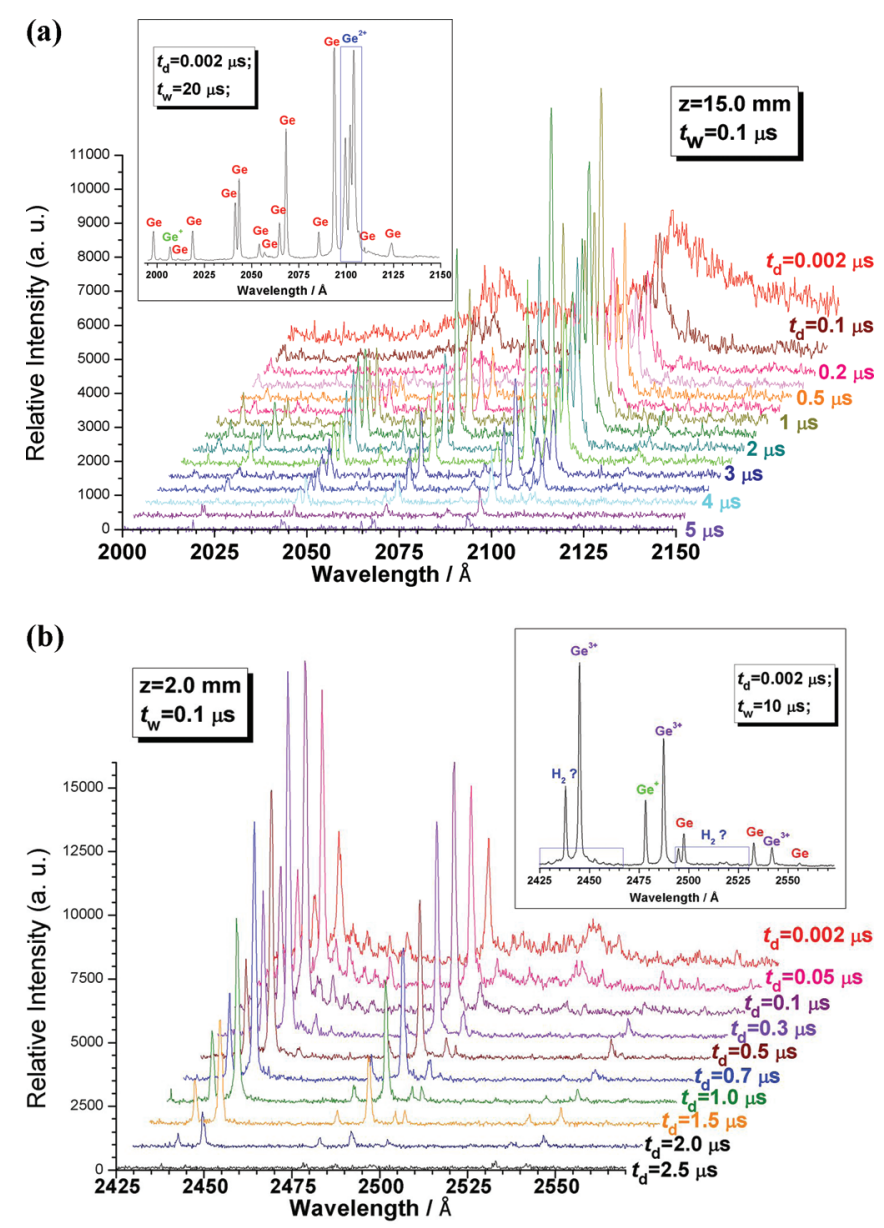

FIG. 3. (Color online) Time-resolved emission spectra from laser-induced $\left(1.4 \mathrm{GW} \mathrm{cm}^{-2}\right)$ Ge plasma observed in two regions at different delay times and a fixed gate width time of $0.1 \mu$ s for (a) $z=15 \mathrm{~mm}$ and (b) $z=2 \mathrm{~mm}$
Time resolved OES measurements can be used to estimate the plasma expansion rate and kinetic energy. The temporal evolution of spectral atomic and ionic line intensities at a constant distance from the target can be used to construct the time-of-flight (TOF) profile. TOF measurements help study the dynamic aspects of LIB plasma. Specifically, this technique gives an indication of the velocity of the emitted species. A coarse estimation of the velocity for the different species in the plasma can be inferred from the time-resolved spectra by plotting the intensities of selected emission lines versus the delay time, and then calculating the velocity by dividing the distance from the target by the time required by the emission to reach the peak $(z / t)$. This method for determination of plasma velocity should be used with care due to the superposition of both expansion and forward movements of the plasma.

Figure 4(a) displays the TOF profiles for germanium breakdown experiments induced by $\mathrm{CO}_{2}$ laser pulses (1.4 $\mathrm{GW} \mathrm{cm}{ }^{-2}$ ). The $\mathrm{Ge}, \mathrm{Ge}^{+}, \mathrm{Ge}^{2+}$, and $\mathrm{Ge}^{3+}$ lines in the $\mathrm{UV}$ region correspond to the spectra of Fig. 3 recorded at $5 \mathrm{~mm}$.
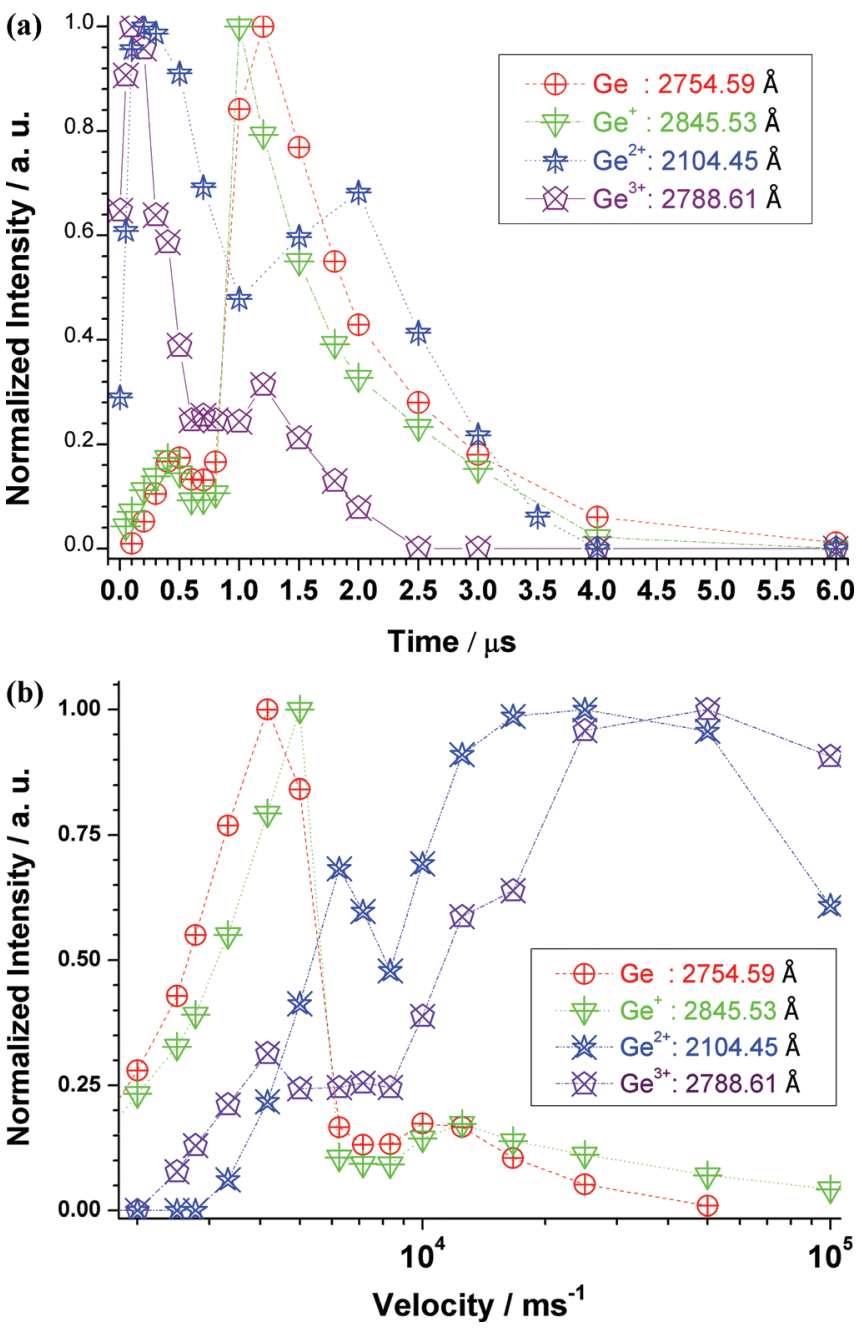

FIG. 4. (Color online) (a) Number density TOF distributions of Ge(2755 $\AA), \mathrm{Ge}^{+}(2845 \AA), \mathrm{Ge}^{2+}(2104 \AA)$, and $\mathrm{Ge}^{3+}(2789 \AA)$ lines as a function of delay time (fixed gate width time of $0.1 \mu \mathrm{s}$ ), for a laser intensity of $1.4 \mathrm{GW}$ $\mathrm{cm}^{-2}$ and $z=5 \mathrm{~mm}$. (b) Velocity distributions derived from the experimental TOF profiles for the indicated species. 
The TOF distributions of $\mathrm{Ge}(2754.59 \AA)$ and $\mathrm{Ge}^{+}(2845.53$ $\AA$ ) species were found to consist approximately of one weak maximum at $\approx 0.5 \mu \mathrm{s}$ and one intense maximum at $\approx 1.2 \mu \mathrm{s}$, respectively. The TOF distributions of $\mathrm{Ge}^{2+}$ were found to consist approximately of one intense maximum at $\approx 0.2 \mu \mathrm{s}$ and one medium maximum at $\approx 1.9 \mu \mathrm{s}$, while that of $\mathrm{Ge}^{3+}$ consists of around one intense maximum at $\approx 0.1 \mu$ s and one weak maximum at $\approx 1.2 \mu$ s. The emission intensity of $\mathrm{Ge}^{3+}$ ionic lines decrease more rapidly than the emission intensity of the other lines. The time duration of different lines of $\mathrm{Ge}$, $\mathrm{Ge}^{+}, \mathrm{Ge}^{2+}$, and $\mathrm{Ge}^{3+}$ were nearly $6,5,4$, and $2.5 \mu$ s, respectively. The evident delay between $\mathrm{Ge}$ and $\mathrm{Ge}^{+}$species, with respect to $\mathrm{Ge}^{2+}$ and $\mathrm{Ge}^{3+}$ ions TOF maxima, is probably due to the formation of these species from the recombination of $\mathrm{Ge}^{2+}$ and $\mathrm{Ge}^{3+}$ ions with electrons.

The velocity distributions that are derived from these TOF distributions are displayed in Fig. 4(b). These distributions present different characteristics. For atomic $\mathrm{Ge}(2754 \AA$ A;

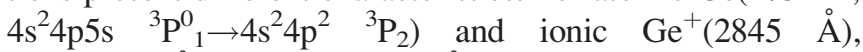
$\mathrm{Ge}^{2+}(2104 \AA)$ and $\mathrm{Ge}^{3+}(2788 \AA)$ species, the velocity distributions are centered at $\approx 4.1,5,25$, and $50 \mathrm{~km} / \mathrm{s}$, respectively. The velocity distributions for $\mathrm{Ge}$ and $\mathrm{Ge}^{+}$are comparatively narrower $[\approx 2.8$ and $\approx 2.7 \mathrm{~km} / \mathrm{s}(\mathrm{FWHM})$, respectively] than the velocity distributions for $\mathrm{Ge}^{2+}[\approx 97 \mathrm{~km} / \mathrm{s}(\mathrm{FWHM})]$ and $\mathrm{Ge}^{3+}[\approx 120 \mathrm{~km} / \mathrm{s}$ (FWHM)].

The different components of the TOF and velocity distributions for each species may be due to different formation mechanisms. Particularly, for $\mathrm{Ge}$ and $\mathrm{Ge}^{+}$species, the complex velocity distribution observed in Fig. 4 might indicate that several processes can be involved in its formation. For example, $\mathrm{Ge}+\mathrm{nh} \nu+\mathrm{e}^{-} \rightarrow \mathrm{Ge}+\mathrm{e}^{-*} \rightarrow \mathrm{Ge}^{+^{*}}+2 \mathrm{e}^{-}$and $\mathrm{Ge}+\mathrm{nh} \nu+\mathrm{e}^{-} \rightarrow \mathrm{Ge}+\mathrm{e}^{-*} \rightarrow \mathrm{Ge}^{*}+\mathrm{e}^{-}$processes (inverse bremsstrahlung and electron-impact ionization and electronic excitation) can be associated to the maxima at $0.5 \mu$ s observed in Fig. 4(a). On the other hand, the observed maxima at $1.2 \mu \mathrm{s}$ can be due to the processes $\mathrm{Ge}^{2+}+2 \mathrm{e}^{-} \rightarrow \mathrm{Ge}^{+}+\mathrm{e}^{-}$and $\mathrm{Ge}^{+}+2 \mathrm{e}^{-} \rightarrow \mathrm{Ge}+\mathrm{e}^{-}$(three body electron-ion recombination). For $\mathrm{Ge}^{2+}$ and $\mathrm{Ge}^{3+}$, two maxima are observed in the TOF profiles. The intense maxima at $\approx 0.2 \mu$ s are probably due to $\mathrm{Ge}+\mathrm{nh} \nu+\mathrm{e}^{-} \rightarrow \mathrm{Ge}+\mathrm{e}^{-*} \rightarrow \mathrm{Ge}^{2+*}+3 \mathrm{e}^{-}$and $\mathrm{Ge}+\mathrm{nh} \nu+\mathrm{e}^{-} \rightarrow \mathrm{Ge}+\mathrm{e}^{-*} \rightarrow \mathrm{Ge}^{3+*}+4 \mathrm{e}^{-}$processes. The medium/weak maxima for $\mathrm{Ge}^{2+}$ and $\mathrm{Ge}^{3+}$ that appear at 1.9 and $1.2 \mu$ s, respectively, could be due to three body electronion recombination processes $\left(\mathrm{Ge}^{3+}+2 \mathrm{e}^{-} \rightarrow \mathrm{Ge}^{2+}+\mathrm{e}^{-}\right.$; $\left.\mathrm{Ge}^{4+}+2 \mathrm{e}^{-} \rightarrow \mathrm{Ge}^{3+}+\mathrm{e}^{-}\right)$. From these observations, it seems that $\mathrm{Ge}^{2+}$ and $\mathrm{Ge}^{3+}$ are mainly generated on the crystalline Ge target while $\mathrm{Ge}$ and $\mathrm{Ge}^{+}$are mainly produced in gas phase at some distance from the laser focal position.

The knowledge of the plasma temperature and electron density is important for understanding the excitation and ionization processes. For plasma in local thermodynamic equilibrium (LTE), the population densities of atomic and ionic electronic states are described by Boltzmann distributions. For LTE plasma, the temperature can be calculated from the relative intensities of several lines by the well-known Boltzmann method. The temperature was obtained from the Boltzmann equation for the line intensities of several Ge lines at a delay time of $3 \mu \mathrm{s}$. The spectral lines wavelengths, energies of the upper levels, statistical weights, and transition probabilities used for these lines were obtained from NIST Atomic Spectra Database. ${ }^{16}$ The obtained excitation temperature was $20000 \pm 1200 \mathrm{~K}$.

The emission spectra reveal noticeable line broadening (see Fig. 3). This broadening has several components, the most important are the instrumental broadening, the Doppler broadening, and the Stark broadening due to the presence of charged species (electrons and ions). This effect is the primary mechanism influencing these spectra. The Doppler broadening (in $\AA$ ) can be obtained as: $\Delta \lambda \lambda_{\mathrm{FWHM}}^{\mathrm{D}}=7.16 \times 10^{-7} \lambda(T / M)^{1 / 2}$, with $\lambda$ being the wavelength, $T$ the temperature in $\mathrm{K}$, and $M$ the atomic mass in amu. In our case $\Delta \lambda_{\text {instrument }}$ was $0.1 \AA$ determined by measuring the FWHM of the $\mathrm{Cu} / \mathrm{Ne}$ lines emitted by a hallow-cathode lamp. The Stark linewidth $\Delta \lambda^{\mathrm{S}}{ }_{\mathrm{FWHM}}$ can be extracted from the measured linewidth $\Delta \lambda_{\text {observed }}$ by subtracting instrumental and Doppler line broadening: $\Delta \lambda_{\text {FWHM }}^{\mathrm{S}}=\Delta \lambda_{\text {observed }}-\Delta \lambda_{\text {instrument }}-\Delta \lambda_{\mathrm{FWHM}}$. The FWHM of the Stark-broadened line $\Delta \lambda_{\mathrm{FWHM}}^{\mathrm{S}}$ is related to the electron density $n_{\mathrm{e}}\left(\right.$ in $\left.\mathrm{cm}^{-3}\right)$ by the approximated expression for a non-H-like line: ${ }^{17}$

$$
\Delta \lambda_{\mathrm{FWHM}}^{\mathrm{S}}=2 W\left(\frac{n_{\mathrm{e}}}{10^{16}}\right),
$$

where $W$ is the electron impact parameter that can be incorporated to different temperatures. For the electron density measurements, we use the widths of the $\mathrm{Ge}^{3+}$ line $\left(5 \mathrm{~d}^{2} \mathrm{D} 5 \mathrm{p}\right.$ ${ }^{2} \mathrm{P}^{0}$ ) at $\approx 2488 \AA$ for delay times $\leq 2.5 \mu$ s and the Ge line $\left(4 \mathrm{~s}^{2} 4 \mathrm{p} 5 \mathrm{~s}{ }^{1} \mathrm{P}^{0}{ }_{1} \rightarrow 4 \mathrm{~s}^{2} 4 \mathrm{p}^{2}{ }^{1} \mathrm{D}_{2}\right)$ at $\approx 3039 \AA$ for delay times $>2.5 \mu$ s. By substituting the Stark line widths of $\mathrm{Ge}^{3+}$ and Ge lines at different delay times in Eq. (1) and the corresponding values of the electron impact parameter, $W$ (0.0240-0.00763 $\AA$ from Popovic et al. ${ }^{18}$ and $0.0140-0.0147$ $\AA$ from Dimitrijevic et al., ${ }^{19}$ respectively, at plasma temperatures between 5000 and $50000 \mathrm{~K}$ ), we obtain the electron density for different delay times.

Figure 5 gives the time evolution of electron density and its first derivative with respect to delay time by setting the gate width of the intensifier at $0.1 \mu \mathrm{s}$ and a laser intensity of $1.42 \mathrm{GW} \mathrm{cm}^{-2}$ and $z=5 \mathrm{~mm}$. The errors provided for electron densities considered the uncertainty in the impact parameters and line broadenings. The initial electron density

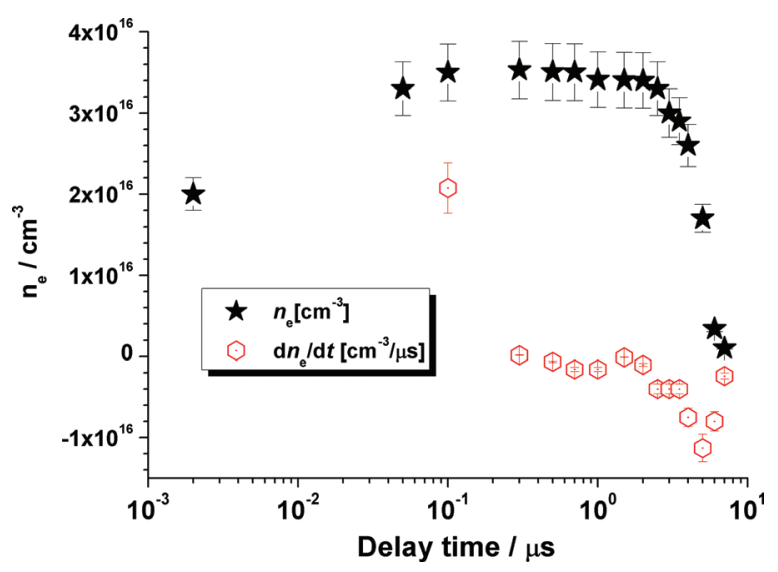

FIG. 5. (Color online) The temporal evolution of electron density $n_{\mathrm{e}}$ and $\mathrm{d} n_{\mathrm{e}} / \mathrm{d} t$ for different delay times from plasma ignition at a laser intensity of $1.42 \mathrm{GW} \mathrm{cm}^{-2}$ and $z=5 \mathrm{~mm}$. 
at $0.002 \mu \mathrm{s}$ is approximately $2 \times 10^{16} \mathrm{~cm}^{-3}$. Afterward, the density increases until $3.5 \times 10^{16} \mathrm{~cm}^{-3}$ over the period of $0.3 \mu \mathrm{s}$ and then decrease as the time is further increased. For times $>2.5 \mu \mathrm{s}$, the electron density decreases quickly. The decrease of $n_{\mathrm{e}}$ is mainly due to recombination between electrons and ions in the plasma. These processes correspond to the so-called radiative recombination and three-body recombination processes in which a third body may be either a heavy particle or an electron. The electron number density $n_{\mathrm{e}}\left(\mathrm{cm}^{-3}\right)$ in the laser induced plasma is governed by the kinetic balance equation ${ }^{20-22}$

$$
\frac{\mathrm{d} n_{\mathrm{e}}}{\mathrm{d} t}=k_{\text {ion }} n_{\mathrm{e}} N_{\mathrm{i}}-k_{\text {rec }} n_{\mathrm{e}}^{3},
$$

where $N_{\mathrm{i}}$ indicates the concentration of heavy particles $\left(\mathrm{cm}^{-3}\right)$, and $k_{\text {ion }}\left(\mathrm{cm}^{3} \mathrm{~s}^{-1}\right)$ and $k_{\text {rec }}\left(\mathrm{cm}^{6} \mathrm{~s}^{-1}\right)$ denote the rate constants of ionization and tree-body electron-ion recombination, respectively. Equation (2) is an approximation of a most general equation that includes several terms corresponding to another loss (diffusion loss, electron attachment, etc) and formation mechanisms. In our experimental conditions, these terms are negligible. In particular, the term due to diffusion loss depends on the focal volume and has a $\Lambda^{-2}$ dependence, with $\Lambda$ being the diffusion length. In our case $\Lambda$ $\approx 21 \times 10^{-3} \mathrm{~cm}$ and therefore, we have a large focal volume. If $\mathrm{d} n_{\mathrm{e}} / \mathrm{d} t=0$, an equilibrium condition can be established; if $\mathrm{d} n_{\mathrm{e}} / \mathrm{d} t \neq 0$, then the ionization $\left(\mathrm{d} n_{\mathrm{e}} / \mathrm{d} t>0\right)$ or the recombination $\left(\mathrm{d} n_{\mathrm{e}} / \mathrm{d} t<0\right)$ prevails and departure from equilibrium occurs. ${ }^{20}$ As is observed in Fig. 5, the equilibrium condition can be established at $0.3 \mu$ s (dynamical equilibrium). For $t<0.3 \mu \mathrm{s}$, the ionization prevails, whereas for $t>0.3 \mu \mathrm{s}$, the three-body electron recombination process dominates over the ionization one. By a nonlinear fit of the intensities for $\mathrm{Ge}^{+}, \mathrm{Ge}^{2+}$, and $\mathrm{Ge}^{3+}$ as function of the delay time to a single exponential decay, we obtained three recombination times of $t_{\text {rec }}=0.91 \pm 0.08 \mu \mathrm{s}, 0.40 \pm 0.08 \mu \mathrm{s}$, and $0.30 \pm 0.07 \mu \mathrm{s}$, respectively. The rate constants of the recombination processes can be estimated from the relation $k_{\mathrm{rec}}=1 /\left(n_{\mathrm{e}}^{2} \cdot t_{\mathrm{rec}}\right)$. The corresponding values for $\mathrm{Ge}^{+}, \mathrm{Ge}^{2+}$, and $\mathrm{Ge}^{3+}$ are $8.9 \times 10^{-28}, 2.0 \times 10^{-27}$, and $2.7 \times 10^{-27} \mathrm{~cm}^{6} \mathrm{~s}^{-1}$, respectively. These values are similar to other literature's reported data. $^{20-22}$

\section{CONCLUSION}

The high density plasma generated by the interaction of a nanosecond $\mathrm{CO}_{2}$ pulsed laser $(10.591 \mu \mathrm{m}, 64 \mathrm{~ns}$, and intensity from 0.27 to $4.9 \mathrm{GW} \mathrm{cm}^{-2}$ ) with $\mathrm{Ge}$ has been investigated using time-integrated and time-resolved OES measurements. The emissions observed are due to electronic relaxation of excited $\mathrm{Ge}, \mathrm{Ge}^{+}, \mathrm{Ge}^{2+}$, and $\mathrm{Ge}^{3+}$. The temperature measurements have been performed by the Boltzmann diagram method using Ge lines, while electron density estimations were made using the Stark broadening method. The main efforts of this work have been concentrated on the study of time-resolved characteristics of the plasma induced by the laser. The velocity distributions for different species were obtained from TOF measurements. The results show faster decays for continuum and $\mathrm{Ge}^{2+}$ and $\mathrm{Ge}^{3+}$ species than for $\mathrm{Ge}$ and $\mathrm{Ge}^{+}$. The plasma for $t_{\mathrm{d}}<3 \mu \mathrm{s}$, at $5 \mathrm{~mm}$ from the focal position, is characterized by high electron and ion densities $\left[(2-3.5) \times 10^{16} \mathrm{~cm}^{-3}\right]$. A delay between $\mathrm{Ge}$ and $\mathrm{Ge}^{+}$ species with respect to $\mathrm{Ge}^{2+}$ and $\mathrm{Ge}^{3+}$ ions TOF maxima is observed. This delay is probably due to the formation of $\mathrm{Ge}$ and $\mathrm{Ge}^{+}$species from the recombination of $\mathrm{Ge}^{2+}$ and $\mathrm{Ge}^{3+}$ ions with electrons.

\section{ACKNOWLEDGMENTS}

We gratefully acknowledge the support received in part by the DGICYT (Spain) Projects: MEC: CTQ2008-05393/ BQU and MEC: CTQ2010-15680/BQU for this research.

${ }^{1}$ Fundamental of Analytical Chemistry, edited by D. A. Skoog and D. M. West, International Edition, 7th Edition (Saunders College Publishing, Philadelphia, Holt, London, 1996).

${ }^{2}$ D. A. Cremers and L. J. Radziemski, Handbook of Laser-Induced Breakdown Spectroscopy (Wiley, New York, 2006).

${ }^{3}$ A. W. Miziolek, V. Palleschi, and I. Schechter, Laser-Induced Breakdown Spectroscopy (Cambridge University Press, Cambridge, UK, 2006).

${ }^{4}$ J. P. Singh and S. N. Thakur, Laser-Induced Breakdown Spectroscopy (Elsevier, New York, 2007).

${ }^{5}$ S. Yamada, S. Oguri, A. Morimoto, T. Shimizu, T. Minamikawa, and Y. Yonezawa, Jpn. J. Appl. Phys. 39, 278 (2000).

${ }^{6}$ J. C. G. Sande, C. N. Afonso, J. L. Escudero, R. Serna, E. Catalina, and E. Bernabeu, Appl. Opt. 31, 6133 (1992).

${ }^{7}$ A. V. Kabashin, V. P. Marien, D. Q. Yang, F. Magny, and M. Meunier, Proc. SPIE 7, 4977C-87 (2003).

${ }^{8}$ A. V. Kabashin, F. Magny, and M. Meunier, J. Appl. Phys. 101, 054311 (2007).

${ }^{9}$ P. J. Wolf, Appl. Phys. A 62, 553 (1996).

${ }^{10}$ B. Nemet and K. Musiol, Contrib. Plasma Phys. 39, 85 (1999).

${ }^{11}$ J. J. Camacho, J. M. L. Poyato, L. Diaz, and M. Santos, J. Appl. Phys. 102, 103302 (2007).

${ }^{12}$ J. J. Camacho, L. Diaz, M. Santos, L. J. Juan, and J. M. L. Poyato J. Appl. Phys. 106, 033306 (2009).

${ }^{13}$ J. J. Camacho, L. Diaz, M. Santos, L. J. Juan, and J. M. L. Poyato J. Appl. Phys. 107, 083306 (2010).

${ }^{14}$ M. Santos, L. Diaz, J. J. Camacho, J. M. L. Poyato, J. Pola, and T. Krenek, Appl. Phys. A 99, 811 (2010).

${ }^{15}$ J. J. Camacho, L. Diaz, M. Santos, and J. M. L. Poyato, Spectrochim. Acta B, 66, 57 (2011).

${ }^{16}$ NIST Atomic Spectra Database online at http://physics.nist.gov/PhysRefData/ASD/index.html, 2008.

${ }^{17}$ H. R. Griem, Principles of Plasma Spectroscopy (Cambridge University Press, Cambridge, UK 1997).

${ }^{18}$ L. C. Popovic and M. S. Dimitrijevic, Bull. Obs. Astron. Belgrade 156, 173 (1997).

${ }^{19}$ M. S. Dimitrijevic, P. Jovanovic, and Z. Simic, Astron. Astrophys. 410, 735 (2003).

${ }^{20}$ M. Capitelli, F. Capitelli, and A. Eletskii, Spectrochim, Acta B 55 (2000) 559-574.

${ }^{21}$ M. Capitelli, A. Casavola, G. Colonna, and A. D. Giacomo, Spectrochim. Acta B 59, 271-289 (2004).

${ }^{22}$ A. D. Giacomo, Spectrochim. Acta B 58, 71-83 (2003). 